\title{
Combining a Hybrid Robotic System with a Bain-Machine Interface for the Rehabilitation of Reaching Movements: A case study with a Stroke Patient*
}

\author{
F. Resquín, J. Ibáñez, J. Gonzalez-Vargas, F. Brunetti, I. Dimbwadyo, S. Alves, L. Carrasco, L. Torres \\ and José Luis Pons.
}

\begin{abstract}
Reaching and grasping are two of the most affected functions after stroke. Hybrid rehabilitation systems combining Functional Electrical Stimulation with Robotic devices have been proposed in the literature to improve rehabilitation outcomes. In this work, we present the combined use of a hybrid robotic system with an EEG-based BrainMachine Interface to detect the user's movement intentions to trigger the assistance. The platform has been tested in a single session with a stroke patient. The results show how the patient could successfully interact with the BMI and command the assistance of the hybrid system with low latencies. Also, the Feedback Error Learning controller implemented in this system could adjust the required FES intensity to perform the task.
\end{abstract}

\section{INTRODUCTION}

Stroke is a leading cause of adult disability around the world. A large number of stroke survivors are left with a unilateral arm or leg paralysis. After completing conventional rehabilitation therapy, a significant number of stroke survivors are left with limited reaching and grasping capabilities [1].

As an alternatively, studies presented in literature have shown the benefits of Functional Electrical Stimulation (FES) for reaching and grasping rehabilitation [2]. FES have many advantages for rehabilitation purposes: it helps the preservation of the muscle mass and functions [3], it increases the muscle excitability, and promotes cortical reorganization [4]. However, due to its non-physiological motor unit recruitment, there are still open challenges that need to be addressed for its use in clinical environments. Similarly, the use of robotic devices was introduced in the last decade to rehabilitate motor functions [5]. In this regard, the advantages offered by theses mechanical devices are exploited to carry out repetitive and intensive tasks with the aim to recover motor functions. Nevertheless, the benefits

\footnotetext{
"This work has been done with the financial support of the Spanish Ministry of Science and Innovation, project HYPER (CSD 2009-00067).

F. Resquín, J. Ibañez, J. Gonzalez-Vargas and J. Pons are with the Neural Rehabilitation Group, of Spanish National Research Council, Madrid, Spain (corresponding author: franresquin@gmail.com).

F. Brunetti is with Catholic University of Asunción, Paraguay.

I. Dimbwadyo, L. Carrasco and L. Torres are with Occupational Thinks Research Group, Centro Superior de Estudios Universitarios La Salle (UAM). Madrid, Spain.

S. Alves is with the Centro de Referencia Estatal de Atención Al Daño Cerebral (CEADAC). Madrid, Spain.
}

offered by these platforms compared to traditional methods are still no completely clear. Therefore, hybrid approaches combining FES with robotic devices have emerged over last years as a way to overcome their individual limitations and to generate a more robust and natural solution for motor assistance and rehabilitation [6].

The design of real-time adaptive controllers is an important factor for the assistance of the hybrid system. The time-varying and non-linear characteristics of the musculoskeletal system must be considered when designing FES-based controllers. Additionally, the short amount of time available in clinical environments limits the design of simple and easy to calibrate controllers.

On the other hand, there is strong evidence that the association between the assistance provided by a rehabilitation device with the user's own movement intentions maximizes rehabilitation outcomes [7]. For this reason, researchers have been focusing on ways to decode volitional commands from physiological signals coming from the central nervous system and relate it with an external assistance.

In this paper, we present preliminary results of a stroke patient using the hybrid robotic platform that we have developed for reaching rehabilitation. The assistance was triggered by volitional commands extracted from an EEGbased brain-machine interface (BMI). A combination of two classifiers was implemented to improve the BMI performance in term of accuracy and latency. Furthermore, the Feedback Error Learning Algorithm (FEL) controller was implemented [8], [9] for the FES controller to adjust the stimuli intensities according to the user's motor responses.

\section{METHODS}

\section{A. Participant}

One male chronic stroke patient (right-handed, 72 years old) was recruited for the experiment. The participant has suffered an ischemic stroke, 3.8 years ago, on his right hemisphere of the brain. The subject had no previous experiences with BCI, FES and robotic devices. The experimental protocol was approved by the Ethical Committee of La Salle University (Madrid, Spain). He signed an informed consent to voluntarily participate in the experiment. 


\section{B. The Hybrid Robotic System}

The development of the hybrid robotic system is based on a distributed architecture, composed of four subsystems: the Assistive Devices (Exoskeleton+FES), Motor Intent Detection, the High-Level Controller (HLC), and the Medical Interface and Visual Feedback. The general overview of the system architecture is shown in Fig. 1a.

The upper limb exoskeleton Armeo Spring® (Hocoma, Switzerland) and the IntFES stimulator (Technalia, Spain) were combined to aid in the execution of the reaching movement. The exoskeleton supports the arm weight against gravity and allows carrying out $3 \mathrm{D}$ reaching movement in an unconstrained space. The electrical stimuli were delivered to the anterior deltoid and triceps muscles using biphasic electrical pulses. This pulse was delivered at a frequency of $40 \mathrm{~Hz}$ with a fixed amplitude (adjusted individually for each muscle before the session) while the FES controller modulated the pulse width to adjust the FES intensity during movement execution

The detection of the motor intentions was carried out by an EEG-based BMI. The BMI was implemented using realtime Simulink (The Mathworks Inc) on a dedicated computer. EEG signals were recorded from 28 positions (AFz, F3-F4, FC3-FC4, C5-C6, CP3-CP4, P3-P4, according to the international $10-10$ system) using active $\mathrm{Ag} / \mathrm{AgCl}$ electrodes (Acticap, Brain Products $\mathrm{GmbH}$, Germany). The reference was set to the voltage of the earlobe contralateral to the affected arm and AFz was used as ground. Additionally, Electromyography (EMG) signals were recorded from two bipolar electrodes placed at anterior deltoid and triceps muscles. EEG and EMG signals were amplified using the gUSBamp (g.Tecgmbh, Austria) and were sampled at 256 $\mathrm{Hz}$.

The HLC generated the joint-specific reference trajectories and guided the subject to perform the movements by regulating the level of assistance (FES intensity) during the rehabilitation exercise. This module was implemented in a PC104 architecture running the xPC Target operation system (Mathwork Inc) for real-time operation.

The medical interface (Fig. 1b) was integrated into the architecture allowing the configuration of the therapy parameters (right/left arm, FES parameters and range of movements). Additionally, a visual feedback module was developed to provide information regarding the arm position (shoulder and elbow). The arm position was represented using 2D figures (Fig. 1c). The current position was depicted using a circle, where the $\mathrm{x}$ - and $\mathrm{y}$-axis indicated the movements of the elbow and shoulder joints, respectively. The system also reported information regarding the quality of the movement by changing the color of the ball after the execution of each trial according to the performance. The color green indicated a good performance, yellow a moderate performance, orange a bad performance and red a poor performance. The Visual Feedback module and the Medical Interface were coded using Matlab as well.

\section{Experiment protocol}

The subject participated in one single session. The study was performed in a sound-attenuated room, where the he sat in a comfortable chair with his affected arm supported by the exoskeleton. The experiment was divided into two phases: measurement and evaluation.
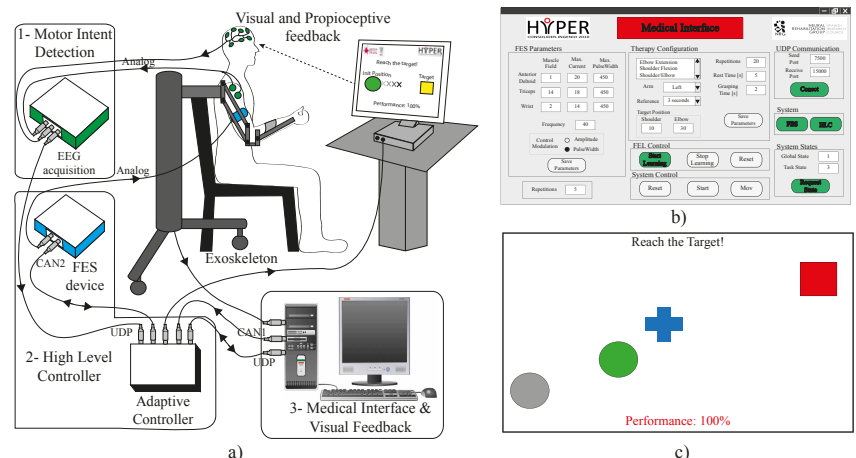

Figure 1. a) General overview of the hybrid robotic system for reaching rehabilitation; b) Medical Interface for therapy parameter configuration; c) Visual and proprioceptive feedback.

During the measurement phase, the subject was instructed to remain relaxed with his eyes open and his gaze fixated on a point on the front screen. He was asked to perform selfinitiated reaching movements with his affected arm. The average distance between movements execution was around 8-15 s. During the resting state in between movements, the subject was asked to remain as relaxed and quite as possible, otherwise, he was asked to start a movement as soon as he felt the urge to do it. The recorded data was used to train an EEG-based detector of the reaching movement onsets. For this purpose, a total of 35 trials were collected for training.

The EMG signals were recorded from the anterior deltoid and triceps muscles, which were used to detect muscle activation events to define the onsets of the reaching movements. The acquired EMG signal was processed offline using a band-pass filter $(55 \geq \mathrm{f} \leq 96 \mathrm{~Hz})$ and a signal rectifier. A single threshold detector was implemented to detect onsets of muscle contractions. The threshold was set as the $7 \%$ of the maximum EMG amplitude during the voluntary movements.

In the second part of the session, the subject was asked to stare at a screen and to repeat the same reaching movement execution. During this process, electrical stimuli and visual feedback were delivered each time the EEG-based detector estimated that a movement intention was detected. The participant was told that the electrical stimuli appeared whenever motor-related mental processes were observed. A total of seven runs of approximately 14 movements were performed, where each trial lasted $2 \mathrm{~s}$.

\section{EEG-Based BMI for User Motor Intention Detection}

We implemented the same method as in [10], to decode the patient's movement intentions based on the EEG signals.

In brief, two classifiers based on the movement-related Event-Related Desynchronization (ERD) and the Bereitschaftspotential (BP) patterns were combined. A naive Bayes classifier was used to detect the ERD patterns preceding the movements. Band-pass filtering (Butterworth, 3th order, $0.5 \mathrm{~Hz}<\mathrm{f} 1,35>\mathrm{f}$ ) ) and Laplacian filter were first applied. The power values were estimated using the Welch's methods in segments of $1.5 \mathrm{~s}$ and for frequencies between 7$30 \mathrm{~Hz}$ (Hamming windows of $1 \mathrm{~s}, 50 \%$ overlapping). The values obtained in the training run between $-3 \mathrm{~s}$ to $-0.5 \mathrm{~s}$ 
(with respect to the movement onsets) were labeled as resting state examples and estimations generated at $\mathrm{t}=0 \mathrm{~s}$ were labeled as movement onset examples. The Bhattacharyya distance was used to select the 10 best features (channel/frequency pairs) to build the Bayesian classifier.

The BP was detected using a finite impulse response lowpass filter (below $2 \mathrm{~Hz}$ ). A virtual channel was obtained by subtracting average potential of channels F3, Fz, F4, C3, C4, $\mathrm{P} 3, \mathrm{Pz}$ and $\mathrm{P} 4$ to channels $\mathrm{Cz}$ and $\mathrm{C} 2$. The average $\mathrm{BP}$ was computed for the resulting channel using the training data and it was used to design a matched filter. To this end, the average BP pattern was extracted from $-1.5 \mathrm{~s}$ to $0 \mathrm{~s}$. During the online function, the matched filter was applied to the virtual channel of the validation dataset.

Finally, outputs from ERD- and BP-based detectors were combined using a logistic regression classifier. Training examples of the resting condition were taken from estimations between $-3 \mathrm{~s}$ and $-0.5 \mathrm{~s}$ with respect to the movement onset. The output estimations at the movement onset were used to model the movement state. The classifier generated estimations of the intention to move every $100 \mathrm{~ms}$. A threshold was applied to the estimations to decide at each moment whether a movement intention was detected. The threshold was optimally obtained using the training dataset and following the criterion of maximizing the TP: percentage of trials with a correct motor intention detection (detection contained in the time interval from $-0.75 \mathrm{~s}$ to $+0.75 \mathrm{~s}$ ) and with no previous incorrect detections.

\section{E. High-Level Controller}

Typically, stroke patients suffer from spasticity that produces an over-activity of flexor muscles of the arm and a loss in activity of triceps, anterior deltoid and fingers extensor muscles [11]. This is why we stimulated the anterior deltoid and triceps muscles to assist shoulder flexion and elbow extension movements.

\section{1) Estimation of the Human Arm Position}

We approximated the position of the human arm joints using the rotation axes of the position transducer embedded in the exoskeleton by considering several assumptions. First, we assumed a parallel arrangement of the human arm with the exoskeleton (Fig. 2a). Second, we assumed that the application of stimulation to the triceps produces a moment about an axis orthogonal to both the forearm and upper arm. Also, that the FES to the anterior deltoid produces a moment about an axis fixed with respect to the shoulder. Therefore, the same bijective transformation used in [12] was implemented to determine the arm axes (shoulder and elbow) and define its position given by vector $\varnothing=\left[\emptyset_{1}, \varnothing_{2}, \varnothing_{3}, \varnothing_{4}\right.$, $\varnothing_{5}$ ] (see Fig. 2a).

\section{2) Feedback Error Learning Controller}

We assumed that the controlled system is composed of two single-input, single-output (SISO) system, thus, the movement of the forearm and upper arm are independent of each other. These considerations allow us to implement two independent Feedback Error Learning (FEL) controllers for each joint, where each one consisted of a proportionalintegral-derivative (PID) feedback controller combined with a neural network (NN) based feedforward loop (see Fig. 2b).
The implemented feedforward loop of the FEL controller allows learning the nonlinear inverse dynamic of the system, while the feedback controller compensates against external disturbances. The NN receives as input the desired kinematic profile and uses the output of the feedback loop as the correction parameter [8]. This learning process is kept along the execution of each movement, where the feedback loop output should tend to zero as learning proceeds.

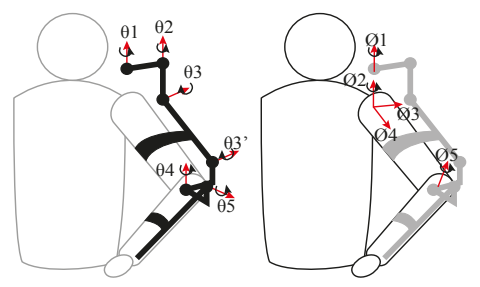

a)

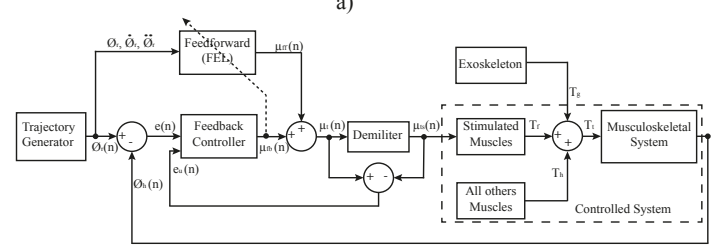

b)

Figure 2. a) Exoskeleton and human arm rotation axes; b) Implemented Feedback Error Learning control scheme.

The NN was defined as a three-layer perceptron network. This network relies on nine input, nine hidden nodes, and one output node. The NN weights were initialized with small random values close to zero, which are updated during movement execution at each sample time.

\section{3) Reference Generator}

To generate the tracking references, we have used the method described by Flash and Hogan [13]. They derived a mathematical expression that describes the way in which the central nervous system move the hand smoothly from one point to another. They called this function the minimum jerk trajectory, and it only requires the target and the duration of the desired position to describe the trajectory completely. In this experiment, we generate the profiles for the position, the velocity and the acceleration, which were used as a reference for the $\mathrm{NN}$ ones the EEG-based BMI trigger the execution of the reaching movement.

\section{F. Metrics Performance}

For BCI validation, we calculated the percent of the good trials. Thus, a good trial was the attempted movements of the subject that produce FES assistance with low latency $(<750$ $\mathrm{ms}$ ). The subject was instructed to say NO when he received the electrical stimuli but he did want to receive it. The HLC validation consists in the analysis of the FEL control signal recorded during the movement execution. For this aim, we used the information of the human arm position and the provided FES intensity.

\section{RESULTS}

Fig. 3 shows the stroke patient during the session. The accuracy of the BMI for detecting the user's movement intentions and the corresponding latency are depicted in Table I. The first row represents the TP in each run and the second row is the average of the detection latency for each run. The accuracy during the first trials is not reported 
because the patient didn't have to report discrepancies between his intentions and the feedback received. Differences were observed in the BCI accuracy across the runs $(5,6,7,8$ and 9), where the performance improved from run 5, and it decreased in run 9. This decayed behavior was attributed to the cognitive fatigue. The average latency in the detections was below $500 \mathrm{~ms}$ for all runs. It is highlighted that negative latencies were not feasible because once the movement intention was detected the stimulation was triggered.

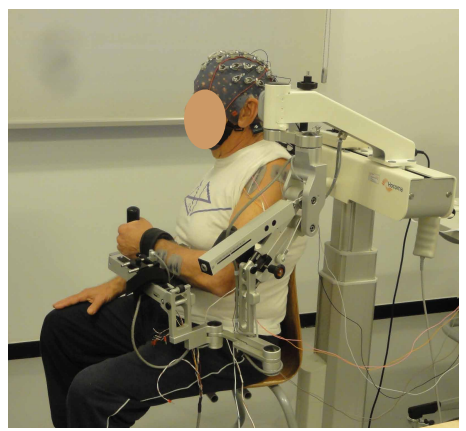

Fig 3. A picture of the patient during the rehabilitation session.

TABLE I. BCI DETECTION ACCURACY AND LATENCY

\begin{tabular}{|l|c|c|c|c|c|}
\hline \multirow{2}{*}{ BCI } & \multicolumn{5}{|c|}{ Training Session } \\
\cline { 2 - 6 } & Run 5 & Run 6 & Run 7 & Run 8 & Run 9 \\
\hline $\begin{array}{l}\text { TP } \\
{[\%]}\end{array}$ & 55.55 & 90.9 & 75.0 & 84.62 & 40 \\
\hline $\begin{array}{l}\text { Latency } \\
{[\mathrm{ms}]}\end{array}$ & $382 \pm 295$ & $334 \pm 290$ & $260 \pm 194$ & $340 \pm 187$ & $346 \pm 196$ \\
\hline
\end{tabular}

Fig. 4 shows the subject's tracking performance. The first row of the figure reveals that, in the first trial of run 1 , the performance was poor with an RMSE $=7.9^{\circ}$ and $37.8^{\circ}$ for shoulder and elbow respectively. After several trials (Fig. 4, second row), the controller learned the inverse dynamic model of the arm, resulting in an overall improvement in tracking accuracy with an RMSE $=5.1^{\circ}$ and $4.3^{\circ}$. Rows 3 and 4 of Fig. 4 show the output of the FEL controller. During the first run, the assistance provided is mainly due the feedback controller $\left(\mathrm{u}_{\mathrm{fb}}\right)$. However, as the controller learned, the FES intensity was given mainly by the feedforward controller $\left(u_{\mathrm{ff}}\right)$. This behavior was more noticeable at the elbow joint.

\section{CONCLUSION AND FUTURE WORKS}

A rehabilitation system that combines mechanical and FES assistance with an EEG-based BMI was presented. A preliminary single session evaluation with one stroke patient was carried out to test the usability of the system. This system represents a first attempt of using the user's own movement intention to trigger the assistance of an upper limb hybrid robotic system by means of BMI. Although the first run the subject experienced some difficulties related the use of the BMI, the patient could successfully command the BMI during the last runs. Typically few sessions are needed to naturally interact with this type of systems. Also, the FEL controller could effectively drive the movement execution by automatically adjusting the FES intensity. Finally, more intervention sessions are needed to validate the proposed BMI-driven hybrid robotic platform and verify its effectiveness in rehabilitation. The future work comprises assessing the system in a clinical intervention with more patients to evaluate it rehabilitation effectiveness.
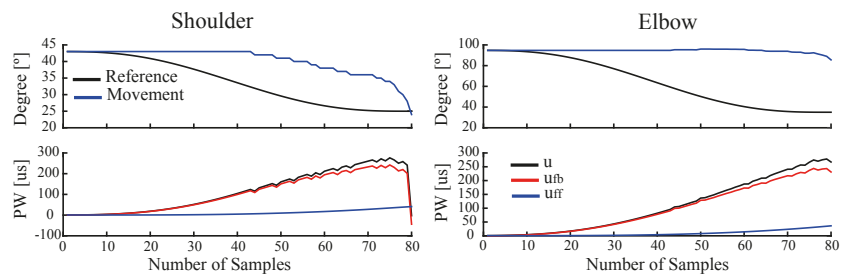

a) Movement 1; Run 1

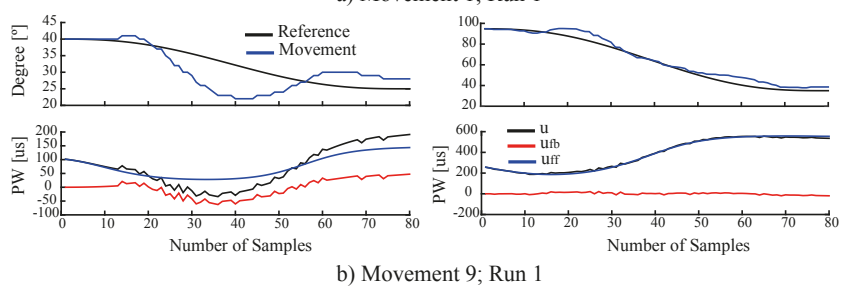

Figure 4. The performance of the FEL controller. a), b) The first row represents the shoulder (left) and elbow (right) movement, while the second row depicts the output of the FEL controller $(u)$, where $u_{f f}$ and $u_{f b}$ represent the feedforward and feedback output respectively.

\section{REFERENCES}

[1] P. Langhorne, F. Coupar, and A. Pollock, "Motor recovery after stroke: a systematic review," Lancet Neurol., vol. 8, no. 8, pp. 741-54, Aug. 2009.

[2] T. A. Thrasher, V. Zivanovic, W. McIlroy, and M. R. Popovic, "Rehabilitation of reaching and grasping function in severe hemiplegic patients using functional electrical stimulation therapy.," Neurorehabil. Neural Repair, vol. 22, pp. 706-714, 2009.

[3] J. Glinsky, L. Harvey, and P. Van Es, "Efficacy of electrical stimulation to increase muscle strength in people with neurological conditions: a systematic review," Physiother. Res. Int., vol. 12, no. 3, pp. $176-1880,2007$.

[4] A. K. Thompson and R. B. Stein, "Short-term effects of functional electrical stimulation on motor-evoked potentials in ankle flexor and extensor muscles," Exp. Brain Res., vol. 159, no. 4, pp. 491-500, 2004.

[5] J. L. Pons, "Rehabilitation exoskeletal robotics. The promise of an emerging field," IEEE Eng. Med. Biol. Mag., vol. 29, no. 3, pp. 57-63, 2010.

[6] J. C. Moreno, A. J. Del-Ama, A. Reyes-Guzmán, Á. Gil-Agudo, R. Ceres, and J. L. Pons, "Neurorobotic and hybrid management of lower limb motor disorders: a review," Med. Biol. Eng. Comput., vol. 49, no. 10, pp. 1119-1130, 2011.

[7] C. Ethier, J. Gallego, and L. Miller, "Brain-controlled neuromuscular stimulation to drive neural plasticity and functional recovery," Curr. Opin. Neurobiol., vol. 33, pp. 95-102, 2015.

[8] M. Kawato, "Feedback-error-learning neural network for supervised motor learning," Adv. neural Comput., vol. 6, no. 3, pp. 365-372, 1990.

[9] Y. Koike, J. Gonzalez, J. Gomez, and W. Yu, "Implementing Feedback Error Learning for FES control," in 2011 4th International Conference on Biomedical Engineering and Informatics (BMEI), 2011.

[10]J. Ibáñez, J. I. Serrano, M. D. Del Castillo, E. Monge-Pereira, F. Molina-Rueda, I. Alguacil-Diego, and J. L. Pons, "Detection of the onset of upper-limb movements based on the combined analysis of changes in the sensorimotor rhythms and slow cortical potentials," $J$. Neural Eng., vol. 11, no. 5, p. 56009, 2014.

[11]P. S. Lum, C. G. Burgar, and P. C. Shor, "Evidence for improved muscle activation patterns after retraining of reaching movements with the MIME robotic system in subjects with post-stroke hemiparesis," Neural Syst. Rehabil. Eng. IEEE Trans., vol. 12, no. 2, pp. 186-194, 2004.

[12]C. T. Freeman, E. Rogers, A. M. Hughes, J. H. Burridge, and K. Meadmore, "Iterative Learning Control in Health Care: Electrical Stimulation and Robotic-Assisted Upper-Limb Stroke Rehabilitation," IEEE Control Syst., vol. 32, no. 1, pp. 18-43, Feb. 2012.

[13]T. Flash and N. Hogan, "The coordination of arm movements: an experimentally confirmed mathematical model.," J. Neurosci., vol. 5, no. 7, pp. 1688-1703, 1985. 